\title{
Research Waste in ME/CFS
}

\author{
Sonia Lee \\ Sydney School of Public Health, University of Sydney, Australia
}

\begin{abstract}
Objective: To compare the prevalence of selective reporting in ME/CFS research areas: psychosocial versus cellular.

Method: A bias appraisal was conducted on three trials (1x psychosocial and $2 \mathrm{x}$ cellular) to compare risk of bias in study design, selection and measurement. The primary outcome compared evidence and justifications in resolving biases by proportions (\%) and ORs (Odds Ratio); the secondary outcome determined the proportion (in \%) of ME/CFS grants at risk of bias.

Results: NS (cellular study) was twice as likely to present evidence in resolving biases over PACE (psychosocial trial) $(\mathrm{OR}=2 \cdot 16 ; 65 \cdot 6 \%$ vs $46.9 \%)$, but this difference was not significant $(p=0 \cdot 13)$. However, NS was five times more likely to justify biases over PACE (OR $=4 \cdot 76 ; 46.9 \%$ vs $15 \cdot 6 \%)$ and this difference was significant $(p=0.0095 ; p<0 \cdot 05)$. PACE was weak in place (operational aspects $32 \%$ ) and NS for data practices (37\%). The proportion of grants were more biased in PACE $(72 \%)$ than NS $(28 \%)$ for evidence, and also more biased in PACE (86\%) than NS (14\%) for justifications.
\end{abstract}

Conclusion: Psychosocial trials on ME/CFS are more likely to engage in selective reporting indicative of research waste than cellular trials. Improvements to place may help reduce these biases, whereas cellular trials may benefit from adopting more translatable data methods. However, these findings are based on two trials. Further risk of bias appraisals are needed to determine the number of trials required to make robust these findings.

Keywords: Research Waste, Selective Reporting, PACE, Chronic Fatigue Syndrome, Myalgic Encephalomyelitis, Clinical Trials, Research Integrity, Evidence-Based Medicine, E-utilities 


\section{Background}

Research waste in clinical trials are seen in outcomes that are not published, or in selective reporting of incidental and spurious findings that cannot be reproduced or translated in practice. When outcomes are not published: resources are wasted, research is stilted, and the study protocol cannot be validated nor repudiated in future protocols. Reviews on publication rates indicate: $50 \%$ of randomised trials are not published (Kasenda et. al., 2014); $88 \%$ for cohort studies (Bogert et al., 2015); and 50\% for pre-clinical and clinical studies (Schmucker et al., 2014). On the other hand, selective reporting is suspected when data is fabricated (intentionally misrepresented); or falsified (intentionally manipulated) in favour of a desired outcome. The potential causes of selective reporting include: poor recruitment, irrelevant endpoints, biased selection criteria and discontinuation, for instance: of 1017 RCTs, $25 \%$ were discontinued, and of those, $9.9 \%$ were discontinued due to poor recruitment (Kasenda et. al., 2014). When outcomes are not published, authors are contacted for missing data in instances of imputing data in unpublished trials (systematic review). However in selective reporting, even if the reported outcomes appear distinctly remarkable: $p$ hacking (extremely good $p$ values); file drawer problem (only positive results), it is difficult to substantiate who is responsible for it, and whether it was intentional; and whether institutional enquiries into research misconduct are worth pursuing if proven to be futile against existing policies, and run the risk of polarising research communities.

As clinical trials become more complex, there is increasing concern selective reporting is harder to detect, and unforeseen complexities may escalate between the oversight bodies that monitor research integrity (eg. issues of research misconduct) versus the autonomy which allow research communities to freely conduct their own research. This review seeks to demonstrate these complexities in Chronic Fatigue Syndrome (CFS).

\section{ME/CFS}

Myalgic Encephalomyelitis (ME) and CFS are not yet considered distinct diagnoses, but have been in the past (White et. al., 2007). The time to onset and the causes of these fatigue-like symptoms are confined to case studies (low evidence), and is still debated among experts. Nevertheless, both are diagnosed when there is an absence of fatigue-related disorders, and 
the patient achieves a minimum threshold score for ME/CFS in at least one fatigue questionnaire eg. The Chalder Fatigue Scale; The Krupp Fatigue Severity Scale; DePaul Symptom Questionnaire etc. (Board on the Health Select Populations, 2015).

In the UK, Cognitive Behavioural Therapy (CBT) and Graded Exercise Therapy (GET) are proposed treatment regimens for ME/CFS to reduce the symptoms of fatigue (NICE guidelines), and are based on the results of a randomised trial (PACE: Pacing, graded Activity, and Cognitive behaviour therapy) on ME/CFS patients $(n=641)$ conducted between March 2005 and November 2008. It recommends 12 to 15 sessions of Cognitive Behavioural Therapy (CBT; Fatigue: $n=161, p=0.0136, p<0.05$ ) over 52 weeks; or 12 to 14 sessions of Graded Exercise Therapy (GET; Fatigue: $\mathrm{n}=159, p$ $=0.0013, p<0.05$ ) over 52 weeks (White et. al., 2011). ME/CFS support groups have rejected this treatment regimen due to harms from post-exertion malaise after GET, and no improvements after CBT. Biomolecular findings further support these claims with evidence of cellular level harms detected after GET (Cook et. al., 2017), and have proposed biomarkers that are unique to ME/CFS (Fluge et. al., 2016). The consensus is that ME/CFS is a complex, multi-faceted disorder that requires a multi-disciplinary approach, and aetiologies at multiple angles, such as: gut microbiota, hormonal, endocrine and immune functions. However, psychosocial angles can also offer important insights when studies are designed based on evidence. The following are lessons learnt from PACE, and recommendations on evidence-based study designs that may facilitate biomolecular studies, and potentially salvage psychosocial perspectives from branching off into research waste.

\section{Methods}

\subsection{Search strategy}

Search terms "myalgic encephalomyelitis", "cognitive", "behaviour", "graded exercised therapy", "adaptive pacing therapy", "gene", "cell", "clinicaltrials.gov" were automatically mined from PubMed using E-utilities on a UNIX platform with no date restriction (fig. 1: sample code). The number of articles, authors and grants were tabulated by year in Excel. All articles, authors, and grants were included, and none were excluded. The search strategy collated MeSH terms for two research trends: Psychosocial versus Cellular. Articles were not scoped (included or excluded) for quality to observe only for research trends, and to minimise selection bias in future studies 
that may choose to replicate this search strategy. This search strategy did not scope for treatment effects as done in systematic reviews, but on research trends in selecting high impact trials for a bias appriasal.

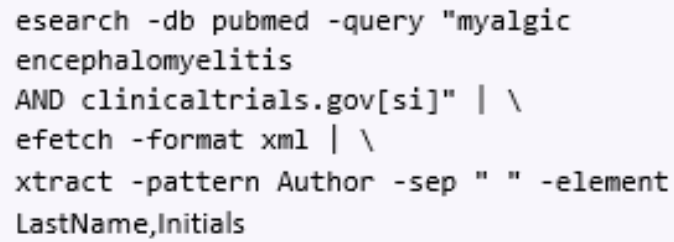

Figure 1: Sample search code in E-utilities (PubMed).

\subsection{Bias appraisal}

The author conducted a bias appraisal (an extended version of Cochrane's bias appraisal tool) on three articles based on search outcomes: year and impact factor (fig. 2): 1. PACE: psychosocial interventions (GET, CBT, and Adaptive Pacing Therapy) to represent psychosocial trends (White et. al., 2011); 2. A neural study (NS) on post-exertion malaise after GET to represent cellular trends (Cook et. al., 2017). 3. A gut study (GS) on profiling gut microbial differences in ME/CFS individuals (Giloteaux et. al., 2017) to also represent cellular trends. In table 1, biases were categorised by: "study design", "selection" and "measurement." Each potential bias was rated by the author with a plus $(+)$ or a minus sign (-) to indicate whether a study presented evidence $(\mathrm{E})$ or a justification $(\mathrm{J})$ for resolving a potential bias. The first two columns E and J rated PACE (White et. al., 2011). The next two columns E and J rated GS (Giloteaux et. al., 2017); followed by ratings for NS (Cook et. al., 2017). The column "Neural Study" offered an example of each rating from Cook and colleagues' (2017) paper. The column "Potential Biases" defined these biases in public health terms. The far right column with the letters "T", "P", "D", "R": Theory (theories and models used in the trial); Place (operational conduct); Recruitment (participant recruitment); Data (data practices) were collated to predict the areas of strengths and weaknesses (selective reporting) in each trial.

\subsection{Statistical analysis}

1. Primary outcome 
bioRxiv preprint doi: https://doi.org/10.1101/133926; this version posted May 7, 2017. The copyright holder for this preprint (which was not certified by peer review) is the author/funder, who has granted bioRxiv a license to display the preprint in perpetuity. It is made available under aCC-BY 4.0 International license.

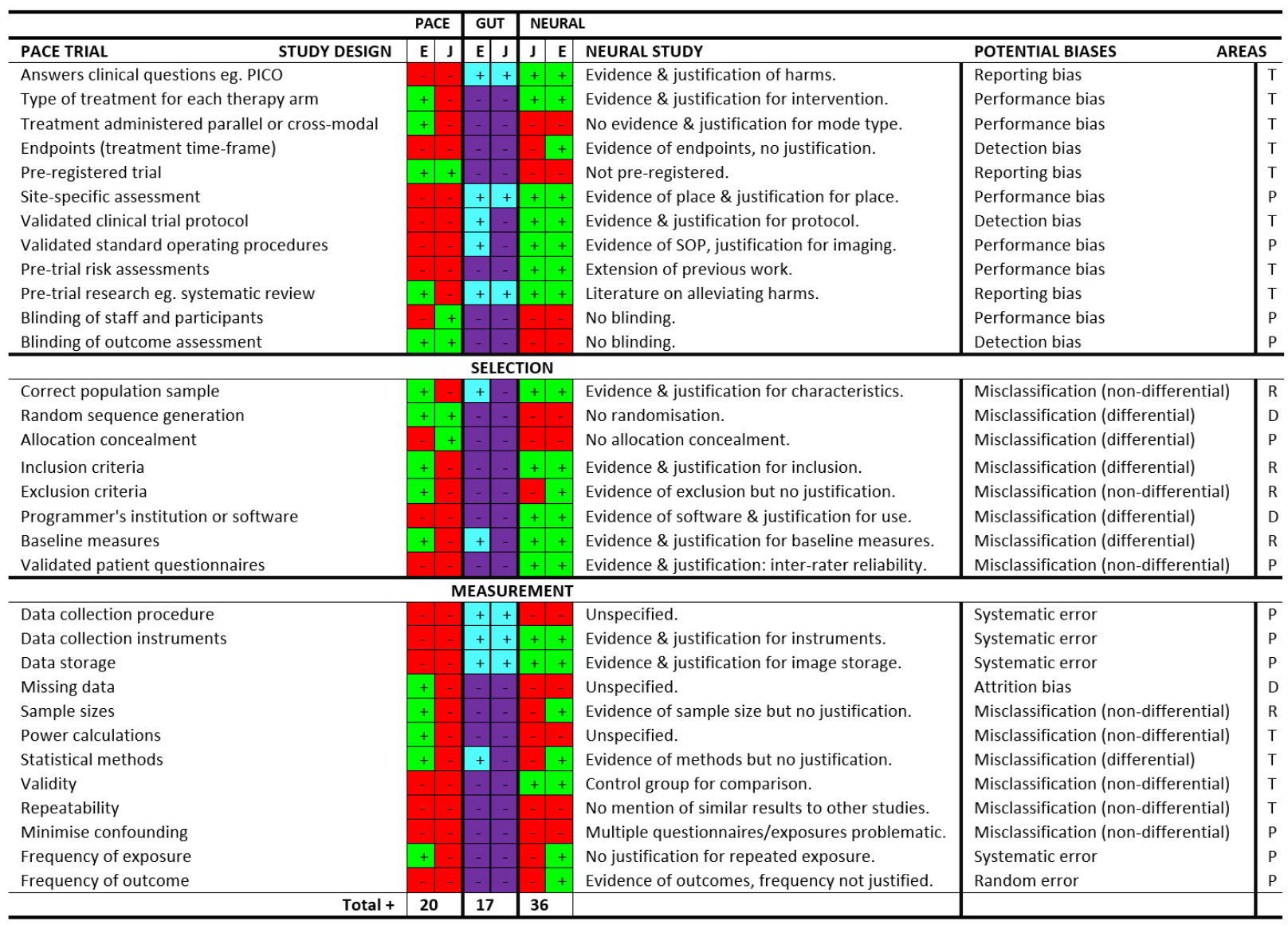

Figure 2: Summary of bias ratings. "+" sign indicates evidence or justifications present in the trial, "." sign indicates it was not present. Areas "T", "P", "D", "R" stand for Theory, Place, Data and Recruitment to represent the potential weak areas in the trial.

ME/CFS research trends: psychosocial or cellular were compared using a $2 \times 2$ contingency table to determine the strength of evidence (table 1 ) and justifications (table 2). Columns $\mathrm{E}$ and $\mathrm{J}$ from the bias appraisal (fig. 2) were tallied and imputed into two contingency tables, and its proportions were compared in deriving the Odds Ratio (OR). The OR determined the strength of evidence or justifications in resolving biases between the two research trends. It also determined the likelihood (in $\%$ ) of evidence or justifications that were present in each research trend. Finally, $Z$ tests (two-tailed) were performed to assess whether the use of evidence or justifications were significantly different between the two 
research trends.

2. Secondary outcome The OR (primary outcome) was applied to the total number of ME/CFS grants (search strategy) declared in each research trend (psychosocial versus cellular) to compare the proportion (in \%) of grants at risk of bias.

3. Software

All data were imputed and analysed by the author using a web-based clinical trials calculator (Centre for Clinical Research and Biostatistics, The Chinese University of Hong Kong) and verified for accuracy in another web-based, effect-size calculator (Campbell Collaboration).

\begin{tabular}{lll}
\hline Evidence & E+ & E- \\
\hline NS & 21 & 11 \\
PACE & 15 & 17
\end{tabular}

Table 1: $2 \times 2$ contingency table comparing evidence tallied from figure 2 (bias appraisal). NS stands for Neural Study; E+ for total plus ratings; E- for total minus ratings.

\begin{tabular}{lll}
\hline Justification & J+ & J- \\
\hline NS & 15 & 17 \\
PACE & 5 & 27 \\
\hline
\end{tabular}

Table 2: $2 \times 2$ contingency table comparing justification tallied from figure 2 (bias appraisal). NS stands for Neural Study; J+ for total plus ratings; J- for total minus ratings.

\section{Results}

The search strategy identified 1750 published articles for psychosocial $\mathrm{ME} / \mathrm{CFS}$ research and 1015 for cellular ME/CFS research between the dates 1951 to 25 March 2017 (day the search was performed). All articles were included in observing research trends: psychosocial versus cellular (fig. 1). Of interest were altmetric scores scoped manually in a psychosocial trial from 2011 (PACE; White et. al., 2011); a cellular study from 2016 (NS; Cook et. al., 2017); and another cellular study from 2016 (GS; Giloteaux et. al., 2016). All articles were selected for popularity (altmetric scores) to represent ME/CFS research trends: 1 . Psychosocial: PACE trial (White et. 


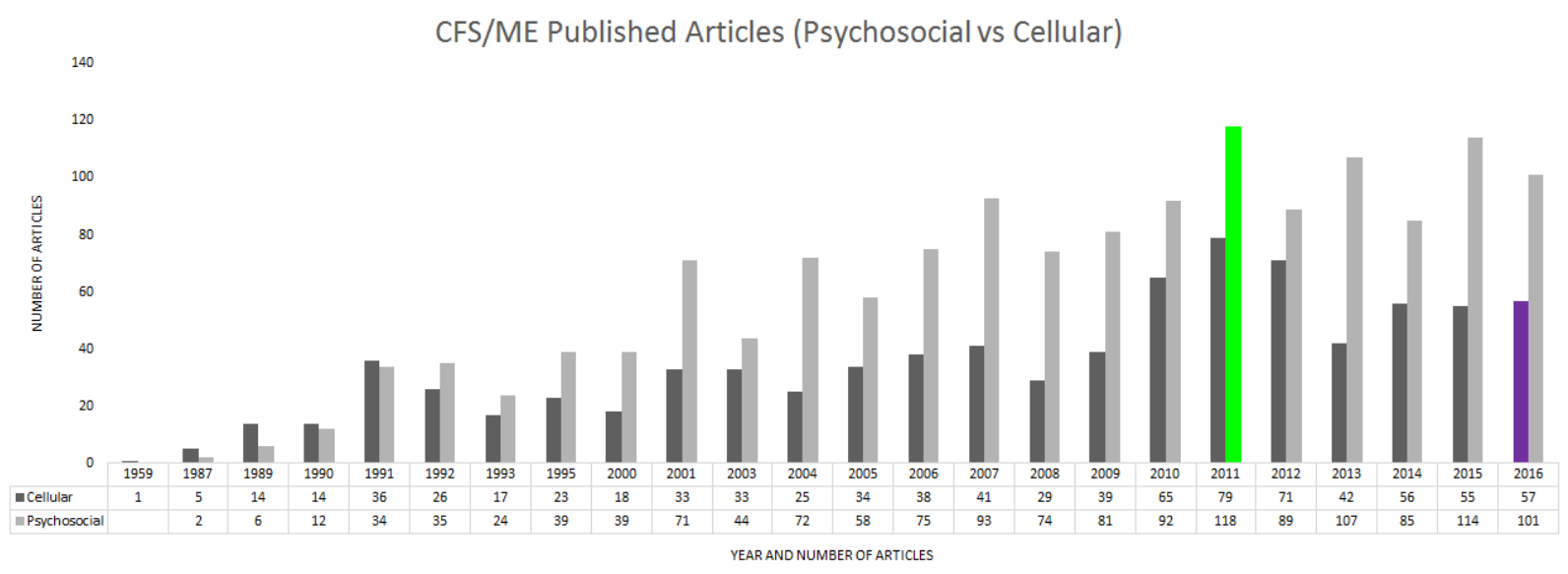

Figure 3: Search results comparing research trends: psychosocial versus cellular by year and number of published articles. The highlighted portions denote which year studies were selected for the bias appraisal.

al., 2011); 2. Cellular: Neural study (Cook et. al., 2017); 3. Cellular: Gut study (Giloteaux et. al., 2016) however, did not undergo any further analysis, since pre-trial conditions (eg. serum samples) from the bias appraisal (table 1) were significantly different in scope to the other two studies $i e$. it did not conform to an intention to treat experimental design.

\subsection{Risk of bias appraisal}

1. Study design

The study design for PACE was unclear, it was neither a randomised controlled trial (no control group) nor a cohort study (randomised); whereas NS had a control group and was likely a case-control study. All of which may have influenced performance biases, attrition biases and other operational biases towards or away from the null. PACE had four treatment arms all ending in 52 weeks but these endpoints were not justified. NS also did not justify measuring neural endpoints in 3 days. Hence both studies were potentially biased on whether treatment effects were measured at an optimal level for a true effect. PACE registered the trial (ISRCTN54285094), NS did not, however, it did specify the site in which the intervention took place, whereas PACE did not, which are potential causes of attrition and reporting biases particularly when trial results can not be verified. NS based the intervention off prior work 
and used objective instruments (imaging scans) for measuring outcomes of an appropriate sample size; PACE on the otherhand, recruited a large sample size for an early phase trial, with no evidence of pre-trial risk assessments on standard operating procedures and clinical trial protocols which normally recruit small sample sizes in early phase trials (so to limit risks on the population of interest). The study design for GS was unclear, it did not conform to an intention to treat experimental design. It had a control group which were matched to cases, but it did not specify the number of specimens, patients and controls recruited by the physician, and if diagnostic tests $i e$. blood draws were done after an already confirmed ME/CFS diagnosis, which are all potential causes of performance, attrition and other biases.

2. Selection bias

NS presented evidence and justifications for population sampling, baseline measures between cases and controls (patient characteristics), and validated questionnaires prior to conducting the study, but it did not conceal treatment allocations despite including a control group. This is highly problematic and can lead to selection biases consistent in case studies without a control group (low evidence). Conversely, if NS used a case-control design, it fell short of blinding and a good coverage of cases obtained from all facilities (not just one) to minimise selection bias. PACE presented better clinical protocols but did not justify population sampling, sample size, selection criteria, baseline measures, questionnaires and software. GS presented very little evidence and justifications in selection eg. it did not specify the number of participants and baseline measures that were relevant to sourcing serum samples. All of which are potential causes of selection bias.

3. Measurement bias

All three studies were scant on evidence and justifications for data collection, analysis and dissemination. PACE did not specify who collected data, the types of software used, or whether the interventions were curative (given there were no treatment endpoints) and relevant to clinical practice. NS did not specify on which specialists assessed the imaging scans and whether the imaging scans were sufficiently sensitive and specific in diagnosing ME/CFS. Also, there was no blinding (if case-control), neither did it specify the number of participants screened per questionnaire (eg. information about lost controls) which often leads to biases in underestimating the prevalence of exposure in 
controls, and overestimate the Odds Ratio in favour of cases (those with ME/CFS). GS presented evidence and justifications for assessing data in a controlled lab environment, but it did not specify any missing data or contaminated samples, any lab errors, confounding or overlapping of RNA sequences with other disease conditions. It also used a rank statistical method, an unspecified machine learning method, a sub-sampling method for validating an unspecified model, all of which were unclear and potential causes of measurement bias.

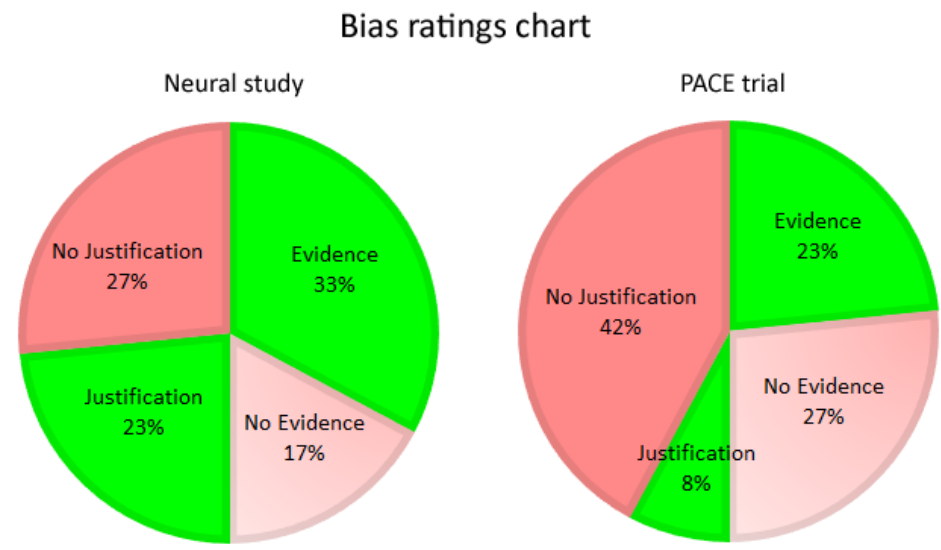

Figure 4: The proportion of bias in evidence and justifications in NS and PACE.

\subsection{Evidence ratings}

NS was twice as likely to be supported by evidence compared to PACE $(\mathrm{OR}=2 \cdot 16)$. NS strength of evidence was at $65 \cdot 6 \%$ and $33 \%$ relative to justifications; for PACE $46.9 \%$ and $23 \%$ relative to justifications (fig. 4). However, there was no significant difference between the two studies in presenting evidence to resolve biases $(p=0 \cdot 13 ; p>0 \cdot 05)$.

\subsection{Justification ratings}

NS was five times more likely to address biases compared to PACE (OR $=4 \cdot 76$ ). Justifications present in NS were at $46.9 \%$ and $23 \%$ relative to evidence; for PACE 15.6\% and $8 \%$ relative to evidence (fig. 4). Unlike evidence, there was a significant difference in presenting justifications to resolve biases in favour of NS $(p=0.0095 ; p<0 \cdot 05)$. 


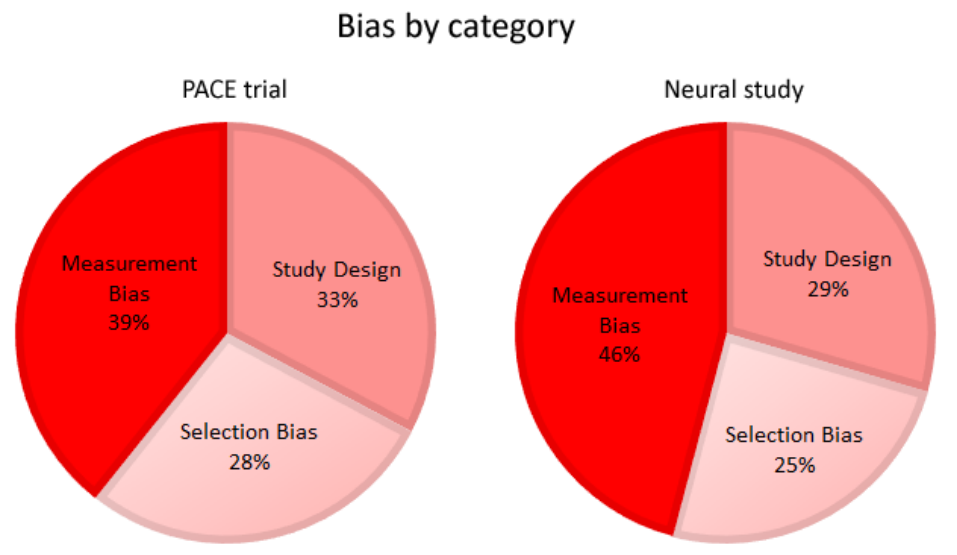

Figure 5: The proportion of bias by category in PACE and NS.

\subsection{Proportion of bias}

Measurement bias was the most prevalent of all biases: PACE (39\%) and NS (46\%); followed by bias in study design: PACE (33\%) and NS (29\%); followed by selection bias: PACE (28\%) and NS (25\%) (fig. 5).

\subsection{Study weaknesses}

PACE was weak in place $(32 \%)$ and theory $(28 \%)$, whereas NS was weak in data (37\%) and place (28\%) (fig. 6).

\subsection{Grants at risk of bias}

The proportion of ME/CFS grants declared in psychosocial publications were at $56 \%$ ( $n=722$; number of articles; search dates 1951 to 25 March $2017)$ and $44 \%(n=568)$ for cellular. The proportion of grants biased in presenting evidence for psychosocial were at $71.8 \%$ and $28.2 \%$ for cellular $(\mathrm{OR}=2 \cdot 16$; fig. 7 ). Grants biased in presenting justifications were at $86 \cdot 4 \%$ for psychosocial and $13 \cdot 6 \%$ for cellular $(\mathrm{OR}=4 \cdot 76$; fig. 7 ).

\section{Discussion}

The results suggest psychosocial ME/CFS trials are more likely to engage in selective reporting than cellular ME/CFS trials. It confirms the concerns raised by ME/CFS groups that psychosocial interventions are harmful, and present questionable therapeutic benefits no different to a placebo (Childs et. 


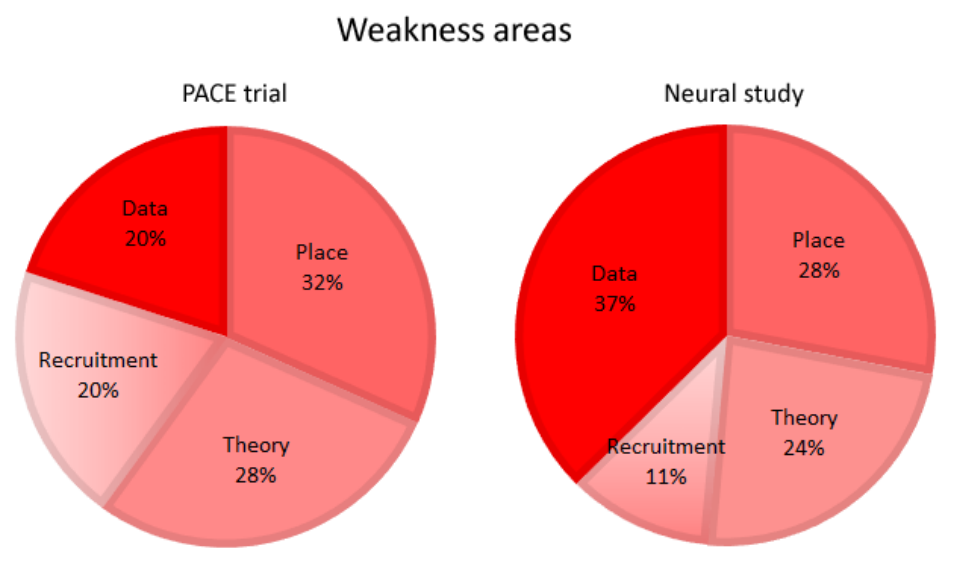

Figure 6: The proportion of weakness areas in PACE and NS.

al., 2015; Lian \& Nettleton, 2015). However, the results also suggest, cellular trials are also likely to engage in selective reporting, but its therapeutic benefit is difficult to assess, since no study as of yet have proposed a therapeutic agent (eg. drug) exclusively designed and marketed for treating ME/CFS (Collatz et. al., 2016). Brurberg and colleagues (2014) propose the need for consistency in ME/CFS research by applying a diagnostic criteria, subject to a systematic evaluation. This need to adequately define ME/CFS is a recurring consensus among researchers (Jason, Boulton \& Friedberg, 2010; Nacul et. al., 2011; Johnston et. al., 2013). Some propose a re-evaluation of domains and criteria in existing patient reported outcome measurements (PROMs) by considering subgroups to account for heterogeneity (different populations) in comorbid conditions (eg. thyroid issues) and patient characteristics (eg. children) (Nacul et., al., 2011; Haywood, Staniszewska \& Chapman, 2012; Johnston et. al., 2014; Haywood, Collin \& Crawley, 2014; Hvidberg et. al., 2015; Murdock et. al., 2016). Others propose the need to investigate biomarkers and immune-mediated networks in developing a prophylactic agent (Fuite, Vernon \& Broderick, 2008; Schlauch et. al., 2016; Vega et. al., 2017; Armstrong et. al., 2017).

If in the latter, cellular trials (eg. biomarkers, immune checkpoints etc.) may benefit from designing outcomes which are sensitive and specific for clinical practice, also safe and reproducible across clinical practice. If this is not feasible, then begin with pre-clinical models (eg. animal models) and confirm risk thresholds (endpoints; safety) to deter heterogeneity (diverse 


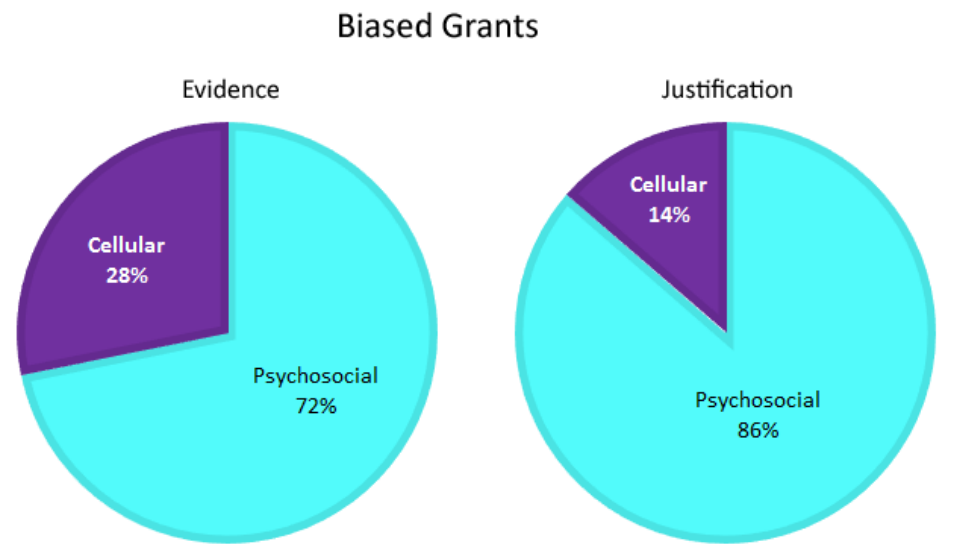

Figure 7: The proportion of bias in ME/CSF grants between research areas: psychosocial and cellular.

or novel methods) in clinical phases from biasing the true effect. On the other hand, psychosocial studies (itemising and validating PROMs), may benefit from ensuring operational aspects (place) are well documented and archived. This ensures selective reporting in measurement- systematic errors and misclassification effects (bias towards or away from the null) can be corrected, and do not misconstrue the true effect.

Selective reporting is a problem in research waste, and a bias appraisal on evidence and justifications is one way to bring light of this. Future studies may look at biases (reoccurring problems) across multiple studies consistent in each research area, so that oversight bodies (eg. grant committees) do not restrict researchers from freely conducting research by enforcing a general standard across all research areas to address research waste.

\section{Acknowledgements}

I dedicate this to ME Awareness Week 2017. I would like to thank ME groups for your passionate advocacy and for sharing your stories with me. It's truly inspiring. Thank you. A special shout out to @postersandme @johnthejack @TweetTipsforME @DrSpeedyandME @MyalgicE @_Lucibee @consent_patient @chrisbrownofca1 @FrancieSaidSo @EllieArnott @JaneEBSmith @velogubbed; @MECFSquestions, @MEMilitant1 and @emmajoy6 for very helpful comments to this manuscript; and @DrFulli for attracting me to this issue. 


\section{Supplementary material}

Link to figures and codes:

http://www.openwetware.org/wiki/User:Sonia_Lee/Notebook/ResearchWasteMECFS

Link to slides:

https://figshare.com/articles/PACE_Trial_Critical_Appraisal_slides_/4685074

\section{References}

Armstrong, C. W., McGregor, N. R., Lewis, D. P., Butt, H. L., \& Gooley, P. R. The association of fecal microbiota and fecal, blood serum and urine metabolites in myalgic encephalomyelitis/chronic fatigue syndrome., Metabolomics, 13(1), 1-13, doi:10.1007/s11306-016-1145-z, 2017.

Brurberg, K. G., Fønhus, M. S., Larun, L., Flottorp, S., \& Malterud, K. Case definitions for chronic fatigue syndrome/myalgic encephalomyelitis (CFS/ME): A systematic review., BMJ Open, 4(2) e003973, doi:10.1136/bmjopen-2013-003973, 2014.

Campbell Collaboration This is a web-based effect-size calculator., Retrieved May 01, 2017 from https://www.campbellcollaboration.org/effect-sizecalculato.html

Childs, N., Robinson, L., Chowdhury, S., Ogden, C., \& Newton, J. L. Consulting patients in setting priorities in myalgic encephalomyelitis (M.E.) research: Findings from a national on-line survey., Research Involvement and Engagement, 1(1), doi:10.1186/s40900-015-0011-x, 2015.

Collatz, A., Johnston, S. C., Staines, D. R., \& Marshall-Gradisnik, S. M. A systematic review of drug therapies for chronic fatigue Syndrome/Myalgic encephalomyelitis., Clinical Therapeutics, 38(6), 12631271.e., doi:10.1016/j.clinthera.2016.04.038, 2016.

Cook, D. B., Light, A. R., Light, K. C., Broderick, G., Shields, M. R., Dougherty, R. J., . . . Vernon, S. D. Neural consequences of postexertion malaise in myalgic Encephalomyelitis/Chronic fatigue syndrome., Brain, Behavior, and Immunity, doi:10.1016/j.bbi.2017.02.009, 2017.

Crawley, E., Mills, N., Hollingworth, W., Deans, Z., Sterne, J. A., Donovan, J. L., . . . Montgomery, A. Comparing specialist medical care with specialist medical care plus the lightning process for chronic fatigue syndrome or myalgic encephalomyelitis (CFS/ME): Study protocol for 
a randomised controlled trial (SMILE trial)., Trials, 14(1), 444-444, doi:10.1186/1745-6215-14-444, 2013.

de Vega, W. C., Herrera, S., Vernon, S. D., \& McGowan, P. O. Epigenetic modifications and glucocorticoid sensitivity in myalgic Encephalomyelitis/Chronic fatigue syndrome (ME/CFS)., BMC Medical Genomics, 10(1), doi:10.1186/s12920-017-0248-3, 2017.

Fluge, Ø, Mella, O., Bruland, O., Risa, K., Dyrstad, S. E., Alme, K., . . . Tronstad, K. J. Metabolic profiling indicates impaired pyruvate dehydrogenase function in myalgic encephalopathy/chronic fatigue syndrome., JCI Insight, 1(21), doi:10.1172/jci.insight.89376, 2016.

Fuite, J., Vernon, S. D., \& Broderick, G. Neuroendocrine and immune network re-modeling in chronic fatigue syndrome: An exploratory analysis., Genomics, 92(6), 393-399, doi:10.1016/j.ygeno.2008.08.008, 2008.

Haywood, K. L., Collin, S. M., \& Crawley, E. Assessing severity of illness and outcomes of treatment in children with chronic fatigue Syndrome/Myalgic encephalomyelitis (CFS/ME): A systematic review of patient-reported outcome measures (PROMs): Systematic review of outcome measures in children with CFS/ME., Child: Care, Health and Development, 40(6), 806-824, doi:10.1111/cch.12135, 2014.

Haywood, K. L., Staniszewska, S., \& Chapman, S. Quality and acceptability of patient-reported outcome measures used in chronic fatigue syndrome/myalgic encephalomyelitis (CFS/ME): A systematic review., Quality of Life Research, 21(1), 35-52, doi:10.1007/s11136-011-9921-8, 2012.

Hvidberg, M. F., Brinth, L. S., Olesen, A. V., Petersen, K. D., \& Ehlers, L. The health-related quality of life for patients with myalgic encephalomyelitis / chronic fatigue syndrome (ME/CFS): E0132421., PLoS One, 10(7), doi:10.1371/journal.pone.0132421, 2015.

Jason, L. A., Boulton, A., Porter, N. S., Jessen, T., Njoku, M. G., \& Friedberg, F. Classification of myalgic Encephalomyelitis/Chronic fatigue syndrome by types of fatigue., Behavioral Medicine, 36(1), 24-31, doi:10.1080/08964280903521370, 2010.

Johnston, S., Brenu, E. W., Staines, D. R., \& Marshall-Gradisnik, S. The adoption of chronic fatigue syndrome/myalgic encephalomyelitis case definitions to assess prevalence: A systematic review., Annals of Epidemiology, 23(6), 371, doi:10.1016/j.annepidem.2013.04.003, 2013.

Johnston, S., Brenu, E. W., Staines, D., \& Marshall-Gradisnik, S. The prevalence of chronic fatigue syndrome/ myalgic en- 
cephalomyelitis: A meta-analysis., Clinical Epidemiology, 5, 105-110, doi:10.2147/CLEP.S39876, 2013.

Johnston, S. C., Brenu, E. W., Hardcastle, S. L., Huth, T. K., Staines, D. R., \& Marshall-Gradisnik, S. M. A comparison of health status in patients meeting alternative definitions for chronic fatigue syndrome/myalgic encephalomyelitis., Health and Quality of Life Outcomes, 12(1), 64, doi:10.1186/1477-7525-12-64, 2014.

Kans, J. Entrez Direct: E-utilities on the UNIX Command Line., Retrieved May 01, 2017 from https://www.ncbi.nlm.nih.gov/books/NBK179288/

Kasenda, B., von Elm, E., You, J., Blmle, A., Tomonaga, Y., Saccilotto, R., . . . Briel, M. Prevalence, characteristics, and publication of discontinued randomized trials., Jama, 311(10), 1045-1052, doi:10.1001/jama.2014.1361, 2014.

Lian, O. S., \& Nettleton, S. United we stand: Framing myalgic encephalomyelitis in a virtual symbolic community., Qualitative Health Research, 25(10), 1383-1394, doi:10.1177/1049732314562893, 2014;2015.

Murdock, K. W., Wang, X. S., Shi, Q., Cleeland, C. S., Fagundes, C. P., \& Vernon, S. D. The utility of patient-reported outcome measures among patients with myalgic encephalomyelitis/chronic fatigue syndrome., Quality of Life Research, 26(4), 913, doi:10.1007/s11136-0161406-3, 2016;2017.

Nacul, L. C., Lacerda, E. M., Campion, P., Pheby, D., Drachler, M. d. L., Leite, J. C., . . . Molokhia, M. The functional status and well being of people with myalgic encephalomyelitis/chronic fatigue syndrome and their carers., BMC Public Health, 11(1), 402-402, doi:10.1186/14712458-11-402, 2011.

Nacul, L. C., Lacerda, E. M., Pheby, D., Campion, P., Molokhia, M., Fayyaz, S., . . . Drachler, M. L. Prevalence of myalgic encephalomyelitis/chronic fatigue syndrome (ME/CFS) in three regions of england: A repeated cross-sectional study in primary care., BMC Medicine, 9(1), 91, doi:10.1186/1741-7015-9-91, 2011.

NICE. Chronic fatigue syndrome/myalgic encephalomyelitis (or encephalopathy): diagnosis and management., Retrieved May 01, 2017, from https://www.nice.org.uk/guidance/cg53/

Schlauch, K. A., Khaiboullina, S. F., De Meirleir, K. L., Rawat, S., Petereit, J., Rizvanov, A. A., . . . Lombardi, V. C. Genome-wide association analysis identifies genetic variations in subjects with myalgic 
encephalomyelitis/chronic fatigue syndrome., Translational Psychiatry, 6, e730, doi:10.1038/tp.2015.208, 2016.

Schmucker, C., Schell, L. K., Portalupi, S., Oeller, P., Cabrera, L., Bassler, D., . . . on behalf of the OPEN consortium. Extent of nonpublication in cohorts of studies approved by research ethics committees or included in trial registries., PloS One, 9(12), e114023, doi:10.1371/journal.pone.0114023, 2014.

The Centre for Clinical Research and Biostatistics (CCRB). The Chinese University of Hong Kong. Retrieved May 01, 2017 from https://www2.ccrb.cuhk.edu.hk/stat/Other.htm

Van Den Bogert, Cornelis A, Souverein, P. C., Brekelmans, C. T. M., Janssen, S. W. J., Van Hunnik, M., Koter, G. H., . . . Bouter, L. M. Occurrence and determinants of selective reporting of clinical drug trials : Design of an inception cohort study., Jama, 311(10), 1045-1052, doi:10.1136/bmjopen-2015-007827, 2014.

White, P., Armstrong, G., Murphy, M., Spencer, P., \& Moss, J. Chronic fatigue syndrome or myalgic encephalomyelitis., BMJ: British Medical Journal, 335(7617), 411-412, doi:10.1136/bmj.39316.472361.80, 2007.

White, P., Goldsmith, K., Johnson, A., Potts, L., Walwyn, R., DeCesare, J., . . Comparison of adaptive pacing therapy, cognitive behaviour therapy, graded exercise therapy, and specialist medical care for chronic fatigue syndrome (PACE): A randomised trial., The Lancet, 377(9768), 823836, doi:10.1016/S0140-6736(11)60096-2, 2011. 